\title{
Effect of Perceptual Factors in the Relationship between a Referent and Objects around the Referent on Young Children's Interpretation of Word Meanings
}

\author{
Takahiro Tamura ${ }^{1}$ \\ ${ }^{1}$ College of Education, Naruto University of Education, Naruto City, Tokushima, Japan \\ Correspondence: Takahiro Tamura, College of Education, Naruto University of Education, Naruto-Cho, Naruto City, \\ Tokushima, 775-8502, Japan.
}

Received: April 7, 2020

Accepted: September 4, 2020

Available online: September 24, 2020

doi:10.11114/ijsss.v8i6.4812

URL: https://doi.org/10.11114/ijsss.v8i6.4812

\begin{abstract}
The purpose of this study was to examine the effect of perceptual factors in the relationship between a referent and objects around the referent on young children's interpretation of word meanings. Participants were 60 children aged 5 years (35 girls, 25 boys; mean age: 5 years 7 months). They participated in a task of interpreting the meaning of nonsense words with high and low inclusiveness conditions. Participants were likely to perceive that objects around the target object included the target object in the high inclusiveness condition. On the other hand, they were not likely to do so in the low inclusiveness condition. As a result, in the high inclusiveness condition, they were likely to speculate that the nonsense label was the name for the target taken together with the object around the target, while in the low inclusiveness condition, they understood it as the name of the target item. The results showed that detailed perceptual factors, such as inclusiveness of the referent and objects around the referent, affected children's language development process.
\end{abstract}

Keywords: language development, vocabulary acquisition, perceptual factors, objects around referents

\section{Introduction}

Children may learn an object's name from people around them through ostensive definition, where the people point to the object and teach its name, "This is an X.". In actual language learning, young children often learn the meaning of new words by ostensive definition. Young children find it difficult to learn the names of objects in this way, because they must identify one correct meaning of X among numerous possibilities (Quine, 1960). Despite this, young children learn word meanings quickly. In order to explain the speed of young children's word acquisition, researchers have pointed out the existence of cognitive constraints and biases that limit the word meanings (Markman \& Hutchinson, 1984; Markman \& Wachtel, 1988; Jones, Smith, \& Landau, 1991). The taxonomic constraint is a cognitive constraint that limits the possibilities of word meaning, as the word for an object names the taxonomic category to which the object belongs (Markman \& Hutchinson, 1984). The whole object assumption is a cognitive constraint whereby a name for an object refers to the whole of it (Markman \& Wachtel, 1988). Mutual exclusivity presupposes that there is only one name for an object (Markman \& Wachtel, 1988). Shape bias is a cognitive bias whereby a word for an object is assumed to apply to other objects similar in shape to that object (Jones et al., 1991). These cognitive constraints and biases have been pointed out to explain the speed of early word learning primarily by cognitive ability as an innate individual factor. Since these cognitive constraints and biases were pointed out, subsequent studies have suggested that various innate individual factors are involved in early word learning.

Bloom (2000) pointed out that young children acquire language through a range of sophisticated cognitive abilities: through general learning and memory abilities, the ability to acquire concepts, and the ability to infer others' intentions. Word learning is therefore not just domain specific. In particular, young children's capacity to infer others' intentions suggests that they utilize various information provided when they learn words, and that their word learning ability is also supported by external factors such as information in the respective situations. Indeed, recent studies have shown that pragmatic cues play an important role in word learning in young children (Grassmann, Stracke, \& Tomasello, 2009; Grassmann \& Tomasello, 2010; Tomasello \& Akhtar, 1995). Although pragmatic clues refer to information derived from people, information provided by the physical situation within which word learning occurs is equally involved in 
young children's word-learning. Smith, Colunga, and Yoshida (2010) suggested that contextual cues play an important role in young children's word-learning. Regarding the effects of contextual cues on word learning, it has been suggested that the space (Samuelson, Smith, Perry, \& Spencer, 2011), background (Meints, Plunkett, Harris, \& Dommock, 2004; Vlach \& Sandhofer, 2011), and position (Benitez \& Smith, 2012) of the referent can influence early word learning. These research findings suggest that the environment and the objects surrounding the referent in word learning situations influence young children's word-learning.

A recent study, clarified that young children have cross-situational learning systems, such as restricting referent words, when various objects are presented multiple times in different situations (Smith \& Yu, 2008; Suanda, Mugwanya, \& Namy, 2014). These results also suggest that young children are extremely sensitive to information provided by objects in situations of word learning. Indeed, it has been founded that the various situations of objects presented in word learning scenes influence the interpretation of word meanings. Perry, Samuelson, Malley, and Schiffer (2010) determined that 18-month-old children are likely to interpret the meanings of nonsense words as being applicable to various objects when presented sets of highly similar exemplars rather than diverse case sets with dissimilar exemplars. Horst, Scott, and Pollard (2010) clarified that 30-month-old children are more likely to correctly interpret the meanings of nonsense words when they are presented with fewer objects that are not the referent of the nonsense word than with many. These findings suggest that when interpreting word meanings, young children are sensitive to the influence of objects surrounding the referent.

Research suggests that this influence is surprisingly strong. In the experiments of Tamura (1997), when a nonsense word was used for an elephant [target item] (a referent for the nonsense word) in a cage, young children were prone to interpret the nonsense word to refer to an elephant in a cage. This suggests that they may speculate the word meanings as including an object such as a cage, that is not a referent, in a situation where objects surround the referent. However, in a similar experiment by Hall and Waxman (1993) in which the interpretation of word meaning was examined in a situation involving objects surrounding the referent (e.g., a bear as the target object was in a car), young children were prone to qualify the word to refer only to a bear that was not restricted by the situation.

What factors are involved in the different results of these studies? There are procedural differences in their experiments. In particular, Hall and Waxman (1993), presented a variety of objects besides the car in which the bear (target object) was positioned, whereas in Tamura' study (1997), only a single object (a cage) surrounded the target object. Specifically, there are differences in terms of the diversity of objects around the referent in the procedures used by their studies Furthermore, the target objects in Hall and Waxman's study (1993) were relatively larger (there was a wide range of objects) than the target objects in Tamura (1997) (only the cage was similar to the target object in size). Tamura (2005) focused on the diversity and dimensions of the objects around the target to examine the effects of these factors on the interpretation of word meanings in 5year olds. Thus, the diversity and dimensions of the objects around the target may explain the disagreement between the results of Hall and Waxman (1993) and Tamura (1997). This result suggests that the relationship between a referent of a word and its surroundings affects participant's interpretation of its meanings. In the real world, a referent of a word exists with various surrounding objects in various situations. In addition, the relationship between the referent and the objects around the referent is also extremely varied. In order to elucidate the mechanism of the interpretation of word meaning in young children in the real world, it is necessary to clarify the nature of the relationship that influences it. In particular, clarifying the kinds of relationships between a referent and objects around the referent by which 5 -year-olds interpret the word meanings as a referent including surrounding objects, we may avoid non-ideal situations that include such relationships when we teach young children the meanings of words by ostensive definition. Additionally, this understanding will ensure the selection of ideal situations in which children are more likely to accurately interpret the words for objects presented to them.

Some studies have examined how the type of relationship between a referent and the objects around it affect the interpretation of word meanings. Tamura (2017) pointed out that the target objects used in the study by Hall et al. (1993) were in low ordinary situations in the relationship to the objects around the targets (e.g., a bear in a car). In contrast, the target object employed in Tamura (1997) had a high level of ordinariness: an elephant in a cage, which suggested that there were different effects on the interpretation of words in young children due to ordinariness. This was examined, but the results failed to confirm the effect of differences between high and low levels of ordinariness of target objects on word meanings in young children, suggesting that their interpretation was not much affected by cognitive factors such as ordinariness that involve qualitative aspects of the relationship between the word referent and the objects around the referent. Furthermore, Tamura (2017) noted that the target and the objects around the target were in high proximity in both Hall et al. (1993) and Tamura (1997), and studied the effect of proximity on word meaning interpretation in young children. They found that 5-year-olds are prone to interpret the word as including surrounding objects in high-proximity situations and excluding the surrounding objects in low-proximity situations. The results indicate that perceptual factors, such as the proximity of a word referent to objects around the referent, influence young children's 
word meaning interpretation. In a real-world language learning situation, the proximity of a referent of the word to its surrounding objects may often be high. Consider these situations, for example: A dog is wearing a collar or is inside a kennel. In these cases, young children may include one of more of the surrounding objects as a referent for the word. However, in children's actual usage of words, errors that include surrounding objects as a referent of a word are not so noticeable. This suggests that there are factors that prevent the inclusion of surrounding objects as a referent of a word even when the referent and the surrounding objects have high proximity. In other words, where the referent and surrounding objects have high proximity, there may be situations where it is easy to include surrounding objects as a referent of a word, but in other situations this may be more difficult. This clarification of the specific levels of proximity would contribute to an avoidance of language learning situations in which young children are likely to include surrounding objects as a referent of a word when being taught the names of objects. Therefore, in this study we examine what kinds of relationships between a referent and its surrounding objects create situations where it is easier or more difficult to include the surrounding objects as referents of the word. We do so specifically in cases where the relationship between a referent of a word and the surrounding objects has high proximity.

The following paragraph examines the target object used in Tamura (2017) in closer detail. In Tamura's (2017) experiments, when an elephant riding in a car or an elephant contained in a cage was presented as a target, young children were likely to include the surrounding objects in the referent of the word. Although the referent and its surrounding objects in these target objects have high proximity, both are considered situations in which the surrounding objects include the referent. This high inclusiveness in the relationship between a word referent and objects around it may be related to Tamura's results (2017). In fact, we refer to a person riding in the vehicle "a passenger," a name that includes the vehicle which is a surrounding object. In this way, if the relationship between a referent of a word and the surrounding objects is one of high inclusiveness, young children might be more likely to interpret the word meaning by including the surrounding objects.

It is thought that perceptual factors are involved in the judgment of whether or not one object includes another object. In fact, some studies have clarified that perceptual factors influence word meaning interpretation in young children. Perry, Samuelson, Malley, and Schiffer (2010) clarifies that there is a difference in young children's interpretation of whether the nonsense words used for the target object (non-solid food) is a material name or a shape name in case the target is made into a nonsense shape or in case it is made into four pieces. In Perry's study, the perceptual factors of the referent itself were manipulated. It has been suggested that the perceptual factors influence the interpretation of word meaning in their study. Tamura (2017) clarifies that there is a difference in young children's interpretation when the referent and its surrounding objects have high proximity, and when they have low proximity. In Tamura's study, the perceptual factors related to the relationship between the referent and objects around the referent were manipulated. It has been suggested that the perceptual factors influence the interpretation of word meaning in their study. In this way, if the perceptual factors related to the reference object itself or the relationship between the reference object and its surroundings influence the interpretation of word meaning in young children, more detailed perceptual factors that require situation judgment may affect children's acquisition of language.

Therefore, in the current study, we studied the effect of inclusiveness in the relationship between the word referent and the objects around the referent on the interpretation of word meanings by young children. Participants chosen were 5-year-old children because they are more sensible to the relationships of a target and objects around the referent than younger children owing to the stage of their perceptual development processes (Zaporozhets, 1965).

If children's interpretation is influenced by inclusiveness in the relationship between a word referent and objects around the referent, they will be prone to interpreting word meaning to include the referent and its surrounding objects only in situations of high inclusiveness rather than in situation of low inclusiveness.

\section{Method}

\subsection{Participants}

Participants were 60 children from public kindergartens in Japan. There were 35 girls and 25 boys among the participants. Their average age was 5 years 7 months, and their age ranged from 5 years 0 months to 5 years 11 months. They belonged to middle-class families who spoke only Japanese. In addition, they had no developmental delays intellectual ability or language ability. Participants were divided into two conditions such that the numbers of girls and boys and their average ages were approximately the same. We obtained permission from the participants' parents for their children to participate in this study. All children voluntarily participated in this study. In relation to the experiment's content and the procedures followed, we observed the ethical principles presented by the Japanese Association of Educational Psychology. Furthermore, we confirmed that the participants' kindergarten teachers and parents did not have any ethical concerns regarding to the experiment's contents and procedures. 


\subsection{Materials}

Two conditions were set for the task of labeling a nonsense word for the target item with the object around the target object. In both conditions, the relationship between the target and the object around the target was one of high proximity. The two conditions were of high inclusiveness and low inclusiveness. The participants under the high inclusiveness condition were presented a situation in which the surrounding object included the target object (an elephant in a cage). Under the low inclusiveness condition, participants were provided a target object in situations where surrounding objects did not include the target object (i.e., an elephant on which a man is riding). To establish the degree of proximity and inclusiveness of the relationship between the target and the object around it under these conditions, we initially tested a convenience sample of 20 university students and measured proximity and inclusiveness using a 5 -step rating scale (the highest score was 5, and the lowest score was 1). The target objects in this study were an elephant or a horse, and university students were asked to rate each. The average rating of the proximity of an elephant with a cage was 4.05 (SD: 0.51), and that of an elephant with a man riding it was 4.15 (SD: 0.55). The average rating of proximity for a horse in a cage and a horse on which a man rides were $4.10(0.36)$ and $4.25(0.44)$, respectively. The average rating of inclusiveness of an elephant with a cage was $4.60(0.50)$, and that of an elephant on which the man rides was $1.53(0.51)$, while the average rating of the inclusiveness of a horse in a cage was $4.55(0.51)$ and that of the inclusiveness of a horse on which a man rides was $1.84(0.50)$. From the results of these rating values, it was concluded that all the target objects were presented in a high proximity situation, while the target objects in a cage have high inclusiveness and those on which a man rides have low inclusiveness. If children's interpretation differs depending on the degree of inclusiveness, it can be inferred that the perceptual factors related to the judgment as to whether or not the referent is included in the surrounding object influence the language acquisition process. Participants in this study were 5-year-old children; therefore, it was necessary to evaluate the recognition of the degree of proximity and inclusion between the target and the object around it in children in this particular age group. Generally, it is thought to be difficult for young children to recognize the degrees of proximity and inclusiveness in such specific situations and to evaluate them using a rating scale. Therefore, only university students used the scale in their rating.

Thirteen choice items were selected to assess participants' interpretation of the meaning of nonsense words used to label targets. The choice items of the high inclusiveness condition were two items of elephants, two items of horses, a crow, a pigeon, two items of an elephant in a cage, two items of a horse in a cage, a pair of scissors, a pencil, and a cage, while the choice items of the low inclusiveness condition were two items of elephants, two items of horses, a crow, a pigeon, two items of an elephant on which a man rides, two items of a horse on which a man rides, a pair of scissors, a pencil, and a man who was not riding an animal. These choice items belong to different conceptual levels (Rosch, 1978). When the target object is an elephant, the elephants of the choice items were the same as the target object in the basic category (elephant); the horses of the choice items were the same as the target object in the superordinate category (mammal); pigeons and crows were the same as the target object in the higher superordinate category (animals); the scissors and pencil were unrelated to the category of animals; and an empty cage or a man not riding on an animal were the objects that surrounded the target objects. Each target object and choice item were a two-dimensional picture drawn with black lines on a $7 \mathrm{~cm} \times 7 \mathrm{~cm}$ white sheet of paper. Two-dimensional pictures were used as target objects and choice items because participants could thus pay attention to only the inclusiveness and the proximity of the target and the object around the target.

\subsection{Procedure}

Participants were allocated to the low or high inclusiveness condition randomly and assessed one by one in a nursery room at a kindergarten.

High inclusiveness condition. The children in the high inclusiveness condition were presented with an elephant in a cage, or a horse in a cage, as a target object, and the experimenter provided the following instruction, "Let's play a game using a foreign language." In this condition, half of the participants were presented with an elephant in a cage and half with a horse in a cage. Figure 1 shows samples of target objects from this condition. Next, the experimenter pointed at the picture of the target object and announced, "Please look at this. This is called a keku (nonsense word) in a foreign language." The experimenter then randomly showed thirteen high inclusiveness choice items individually and asked, "Do you think that this is called a keku in a foreign language?" Participants were asked to answer "Yes" or "No". The order in which the choice items were showed was different for each child. 


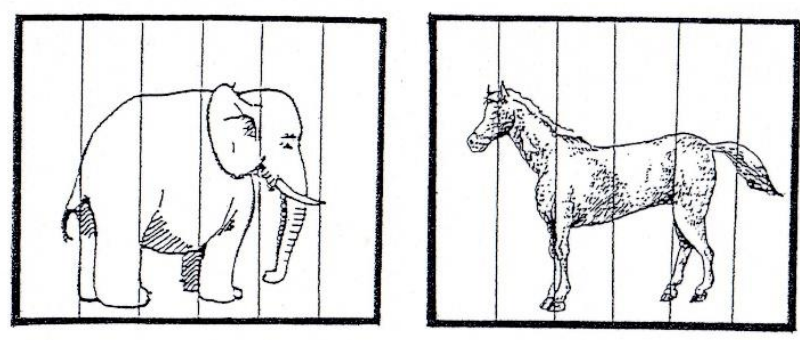

Figure 1. Examples of target objects under the high inclusiveness condition

Low inclusiveness condition. Participants in this condition were presented with either an elephant on which a man was riding or a horse on which a man was riding as the target object. Figure 2 shows samples of target objects from this condition. In addition, they were presented with thirteen choice low inclusiveness items. Except for the content of these presentation objects, the procedure followed was the same as that of the high inclusiveness condition.
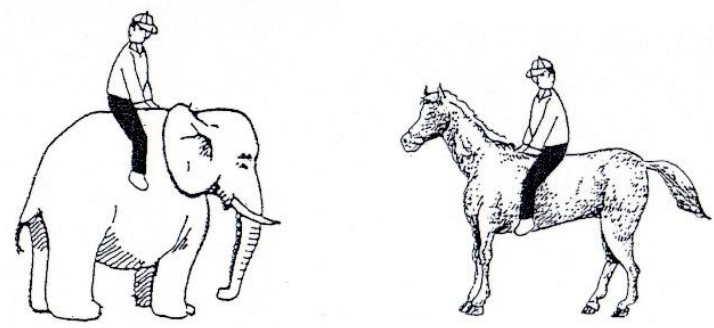

Figure 2. Examples of target objects under the low inclusiveness condition

Figure 3 shows examples of all choice items under the high inclusiveness condition, and Figure 4 shows examples of choice items of objects with surrounding objects under the low inclusiveness.

\subsection{Analysis}

We classified participants' responses into the following three patterns: (1) the Object Name pattern that refers to a response pattern in which, irrespective of the absence or presence of surrounding objects, all elephants or all horses were chosen, and the other objects were not chosen at all; (2) the Name Restricted Surrounding Situation pattern that refers to a response pattern in which all choice items of an elephant, or a horse in a cage, or an elephant, or a horse on which a man was riding were chosen, but none of the other items were chosen; and (3) the Other pattern, in which answers other than the above two patterns were provided. Participants whose responses fell in the Object Name pattern speculated that the nonsense word was "an elephant" or "a horse." The influence of inclusiveness between the target object and the object around the target on the interpretation of the nonsense word was examined, and Fisher's exact test was carried out to examine differences in the number of participants classified into each pattern. When testing a pattern, the number of participants in other patterns were considered included.

\section{Results and Discussion}

Table 1 indicates the number and percentage of participants who displayed each reaction pattern under the high and low inclusiveness conditions. In order to compare the differences between the number of participants displaying each reaction pattern in each condition, Fisher's exact test was carried out. As a result, the number of the Object Name pattern was significantly lower in the high inclusiveness condition than in the low inclusiveness condition $(\mathrm{p}=.033)$. Conversely, the number of the Name Restricted Surrounding Situation pattern was significantly lower in the low inclusiveness condition than in the high inclusiveness condition $(\mathrm{p}=.038)$. For the Other pattern, there was no significant difference between the high and low inclusiveness condition. These results show that in situations where the referent of the word is included in its surrounding objects, young children are 

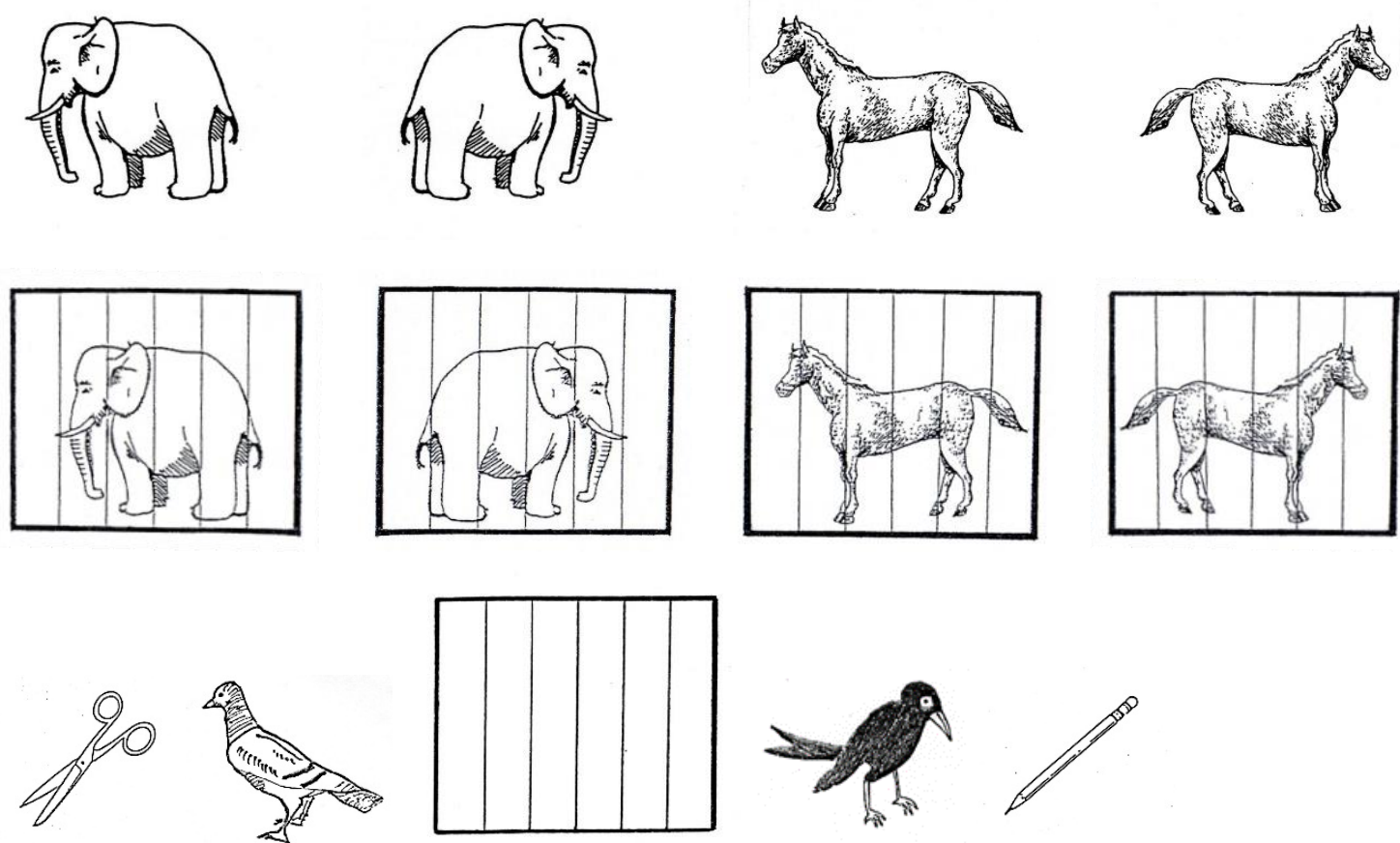

Figure 3. Examples of all choice items under the high inclusiveness condition
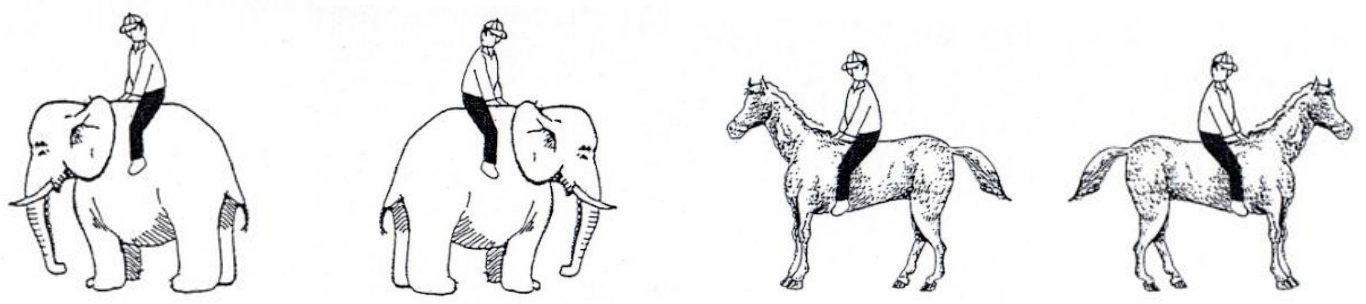

Figure 4. Examples of choice items of objects with surrounding objects under the low inclusiveness condition

Table 1. Number and Percentage of Children in Each Condition Stratified According to Response Pattern

\begin{tabular}{lrrrrc}
\hline & \multicolumn{3}{c}{ High Inclusiveness } & \multicolumn{2}{c}{ Low Inclusiveness } \\
\multicolumn{1}{c}{ Pattern } & $\mathrm{n}$ & $\%$ & $\mathrm{n}$ & $\%$ \\
\hline Object Name & 7 & 23.3 & 16 & 53.3 \\
Name Restricted Surrounding Situation & 19 & 63.3 & 10 & 33.3 \\
Other & 4 & 15.4 & 4 & 15.4 \\
\hline
\end{tabular}

likely to interpret the word meaning as including the surrounding objects. On the other hand, in situations where the referent of the word does not include its surrounding objects, they are likely to interpret the word meaning as not including the surrounding objects. Both high and low inclusiveness conditions in this study had a high degree of proximity between the referent object of the word and its surrounding objects. Nevertheless, in situations where the referent of the word did not include the surrounding objects, the young children interpreted the meaning of the word as the referent without including the objects around the referent. This result suggests that the proximity between the referent of the word and the objects around the referent is not the only factor that is involved in young children's interpretation of the word meaning as including the surrounding objects. In other words, even in situations where the referent of the word and its surrounding 
objects are in high proximity to each other, or in a situation where the referent of the word is included in the surrounding objects, young children are likely to interpret the word meaning as including surrounding objects. Tamura (2017) pointed out that proximity between the referent of a word and the objects around the referent influenced the word meaning interpretation. However, given that the target objects used in this experiment were included in their surrounding objects, this may have affected the results. The results of this study revealed that the factors related to a more detailed perceptual relationship, such as inclusiveness between the referent of the word and the objects around the referent, affect the word meaning interpretation in 5-year-olds. The fact that this factor is present in young children's interpretation of word meanings suggests that a more detailed perception, which includes a situational judgment as to whether or not the surrounding object includes the referent, is involved in word learning in young children, rather than a relatively simple perception such as proximity between the word referent and the objects around it.

In the real world, it is common for the referent object of a word to exist in a situation with high proximity to its surrounding objects. For example, when a dog is the referent object, there are situations where the dog is wearing a collar or walking with its owner. However, young children are not likely to interpret the word meaning by including these surrounding objects in the referent of the word "dog". It may be that there are not that many situations where surrounding objects completely include the referent objects of a word. Nonetheless, like tigers in a zoo, there are cases where referent objects of a word are always included in its surrounding objects ("a cage" in this case). In this case, young children are likely to guess the meaning of "a tiger" by including the object around it "a cage". To avoid such misinterpretation of a word's meaning, it might be necessary to distinguish a referent object from its surrounding objects such as, "There is a tiger in the cage. That is a tiger."

Previous studies of word learning have revealed that contextual factors such as the space (Samuelson et al., 2011), background (Meints et al., 2004; Valch \& Sandhofer, 2011), and position (Benitez \& Smith, 2012) of the referent may influence word learning in young children. Since it is thought that these contexts are discriminated by our perception, it can be said that perceptual factors in the language learning situation have a great influence on word learning in young children. The results of the current study suggest that more detailed perceptual factors such as whether surrounding objects included referent objects of a word, influenced word learning in young children. This suggests that the interpretation of word meaning is through more advanced cognitive processes such as interpreting perceptual situations than grammar learning in which certain rules are learned. When adults teach an object's name to young children, it is thus necessary to consider the influence of such detailed perceptual factors and young children's advanced cognitive processes.

This study has several limitations. In the experimental material of this study, the surrounding objects were limited to a cage and a man, showing high and low inclusiveness with the referent of the word. However, we cannot rule out the possibility that the results of this study reflect particular effects within each set of surrounding objects. Future research should investigate this issue using a variety of other surrounding objects with high and low inclusiveness with the referent to confirm carefully the influence of inclusiveness between the referent and its surrounding objects. Moreover, the surrounding objects in our experiment were from two different categories, both artifacts (a cage) and natural (a man). It may be that the results of this study reflect the differences in these types of surrounding objects. Further research should examine the effects of pure inclusiveness by using surrounding high and low inclusiveness objects of the same type.

In this study, we explored the influence of detailed perceptual factors such as inclusiveness between a word referent and the objects around it in the language learning of 5-year-old children. If such detailed perceptual factors influence young children's word learning, it is likely that a variety of other perceptual factors in the relationship between the referent and the objects around the referent may contribute to word learning, such as when surrounding objects are part of the referent (e.g., horns growing from a deer's head), are worn on the referent (e.g., a collar attached to a dog), or are held by the referent (e.g., a banana held by a monkey). Future research should systematically examine the influence of these more detailed perceptual factors.

\section{References}

Benitez, V. L., \& Smith, L. B. (2012). Predictable locations aid early object name learning. Cognition, 125, 339-352. https://doi.org/10.1016/j.cognition.2012.08.006

Bloom, P. (2000). How children learn the meaning of words. Cambridge, MA: MIT Press. https://doi.org/10.7551/mitpress/3577.001.0001

Grassmann, S., \& Tomasello, M. (2010). Young children follow pointing over words in interpreting acts of reference. Developmental Science, 13(1), 252-263. https://doi.org/10.1111/j.1467-7687.2009.00871.x

Grassmann, S., Stracke, M., \& Tomasello, M. (2009). Two-year-olds exclude novel objects as potential referents of novel 
words based on pragmatics. Cognition, 112(3), 488-493. https://doi.org/10.1016/j.cognition.2009.06.010

Hall, D. J., \& Waxman, S. (1993). Assumptions about word meaning: Individuation and basic-level kinds. Child Development, 64, 1550-1570. https://doi.org/10.2307/1131552

Horst, J. S., Scott, E. J., \& Pollard, J. A. (2010). The role of competition in word learning via referent selection. Developmental Science, 13(5), 706-713. https://doi.org/10.1111/j.1467-7687.2009.00926.x

Jones, S. S., Smith, L. B., \& Landau, B. (1991) Object properties and knowledge in early lexical learning. Child Development, 62, 499-516. https://doi.org/10.2307/1131126

Markman, E. M., \& Hutchinson, J. E. (1984). Children's sensitivity to constraints on word meaning: Taxonomic versus thematic relation. Cognitive Psychology, 16, 1-27. https://doi.org/10.1016/0010-0285(84)90002-1

Markman, E. M., \& Wachtel, G. F. (1988). Children's use of mutual exclusivity to constrain the meanings of words. Cognitive Psychology, 20, 121-157. https://doi.org/10.1016/0010-0285(88)90017-5

Meints, K., Plunkett, K., Harris, P. L., \& Dimmock, D. (2004). The cow on the high street: Effects of background context on early naming. Cognitive Development, 19(3), 275-290. https://doi.org/10.1016/j.cogdev.2004.03.004

Perry, L. K., Samuelson, L. K., Malley, L. M., \& Schiffer, R. N. (2010). Learn locally, think globally: Exemplar variability supports higher-order generalization and word learning. Psychological Science, 21(12), 1894-1902. https://doi.org/10.1177/0956797610389189

Quine, W. V. O. (1960). Words and objects. Cambridge, MA: MIT Press.

Rosch, E. (1978). Principles of categorization. In E. Rosch \& B. B. Lloyd (Eds.), Cognition and categorization. Hillsdale, NJ: Erlbaum, pp. 27-48.

Samuelson, L. K., Smith, L. B., Perry, L. K., \& Spencer, J. P. (2011). Grounding word learning in space. PloS One, 6 (12), e28095. https://doi.org/10.1371/journal.pone.0028095

Smith, L. B., \& Yu, C. (2008). Infants rapidly learn word-referent mapping via cross-situational statistics. Cognition, 106, 1558-1568. https://doi.org/10.1016/j.cognition.2007.06.010

Smith, L. B., Colunga, E., \& Yoshida, H. (2010). Knowledge as process: Contextually cued attention and early word learning. Cognitive Science, 34, 1287-1314. https://doi.org/10.1111/j.1551-6709.2010.01130.x

Suanda, S. H., Mugwanya, N., \& Namy, L. L. (2014). Cross-situational statistical word learning in young children. Journal of Experimental Child Psychology, 126, 395-411. https://doi.org/10.1016/j.jecp.2014.06.003

Tamura, T. (1997). Youji no goimigentei ni oyobosu jirei no syuuhenjyokyo no kouka. [Effects of object's surrounding situation on qualifying word meaning in young children.] Kyouikushinrigakukenkyu. [Japanese Journal of Educational Psychology], 45, 474-481. [in Japanese with English abstract] https://doi.org/10.5926/jjep1953.45.4_474

Tamura, T. (2005). Youji no goimigentei ni oyobosu syuhenjibutsu no tayousei to soutaitekiokisa no eikyo. [The effects of the diversity and relative dimension of the peripheral objects on the meanings of the qualifying word for young children.] Sinrigakukenkyu. [The Japanese Journal of Psychology], 75, 487-494. [in Japanese with English abstract] https://doi.org/10.4992/jjpsy.75.487

Tamura, T. (2017). Effect of objects surrounding a referent on young children's interpretation of word meanings. International Journal of Social Science Studies, 5(1), 54-62. https://doi.org/10.11114/ijsss.v5i1.1994

Tomasello, M., \& Akhtar, N. (1995). Two-year-olds use pragmatic cue to differentiate reference to objects and actions. Cognitive Development, 10, 201-224. https://doi.org/10.1016/0885-2014(95)90009-8

Vlach, H. A., \& Sandhofer, C. M. (2011). Developmental differences in children's context-dependent word learning. Journal of Experimental Child Psychology, 108(2), 394-401. https://doi.org/10.1016/j.jecp.2010.09.011

Zaporozhets, A. V. (1965). The development of perception in the preschool child. In P.H. Mussen (Ed.), European research in cognitive development (Monographs of the Society for Research in Child Development, Vol. 30), (pp. 82-101.) https://doi.org/10.2307/1165778

\section{Copyrights}

Copyright for this article is retained by the author(s), with first publication rights granted to the journal.

This is an open-access article distributed under the terms and conditions of the Creative Commons Attribution license which permits unrestricted use, distribution, and reproduction in any medium, provided the original work is properly cited. 